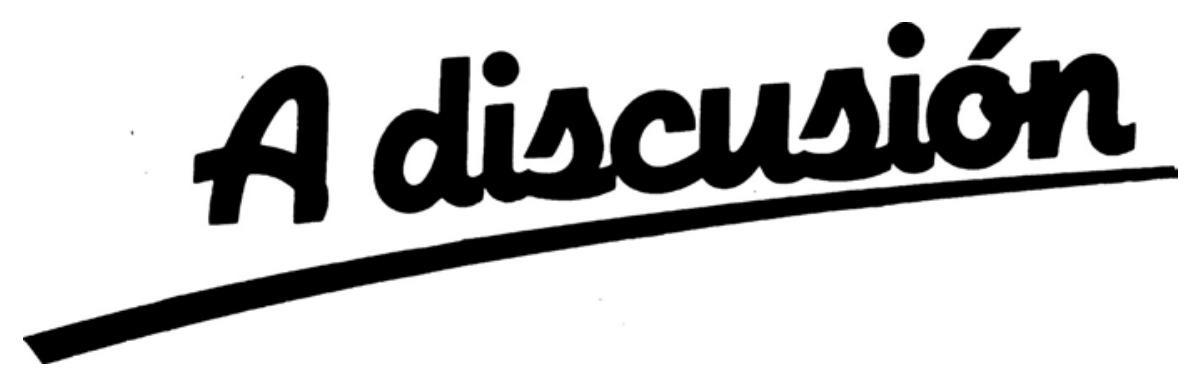

\title{
OPPORTUNITY ANALYSIS OF NEWBORN SCREENING PROGRAMS*
}

\section{Carmen Herrero and Juan D. Moreno-Ternero**}

WP-AD 2005-02

Corresponding author: J.D. Moreno: Yale University. Department of Political Science. P.O. Box 208301. New Haven, CT 06520-8301. USA. Phone: 203.432.3948. Fax: 203.432.6196. e-mail: juande@merlin.fae.ua.es.

Editor: Instituto Valenciano de Investigaciones Económicas, S.A.

Primera Edición Enero 2005

Depósito Legal: V-443-2005

IVIE working papers offer in advance the results of economic research under way in order to encourage a discussion process before sending them to scientific journals for their final publication.

* We thank Marco Mariotti for helpful comments and suggestions. Moreno-Ternero's research was possible thanks to a post-doctoral fellowship from the Generalitat Valenciana. Financial support from the Instituto Valenciano de Investigaciones Económicas (IVIE), from the Ministerio de Ciencia y Tecnología, under project BEC2001-0535, from the Generalitat Valenciana, under project GRUPOS 03-086 from the Fundación BBVA, under project 1-04X, is also gratefully acknowledged.

** C. Herrero: Universidad de Alicante, Dpto. Fundamentos de Análisis Económico; J.D. Moreno: Yale University. 


\title{
OPPORTUNITY ANALYSIS OF NEWBORN SCREENING PROGRAMS
}

\section{Carmen Herrero and Juan D. Moreno-Ternero}

\begin{abstract}
There exist congenital diseases that reduce newborns' potential opportunities. This reduction is sometimes alleviated if the congenital disease is early detected thanks to a newborn screening program. We propose an outcome measurement of newborn screening programs based on the opportunity gains they offer after its implementation. We show that, under plausible assumptions, preferences among the available screening programs for a particular disease according to this new outcome measurement, do not depend on the metric of opportunity. We also apply our model to the current debate about choosing between a selective or a universal newborn hearing screening program to detect congenital hearing impairment.
\end{abstract}

JEL Classification: D61, D63, I12, I18

Key words: Opportunity analysis, potential success, screening programs. 


\section{Introduction}

The discipline of health economics is becoming enormously popular nowadays. The economic evaluation directs many types of health care decisions like, for instance, the selection of a clinical strategy for a given condition out of a set of alternatives. A related problem is the one we endorse here.

One of the most flourishing areas within health economics is the one that concerns the equity in the delivery of health care (e.g., Bleichrodt (1997), van Doorslaer et al., (2000), Wagstaff and van Doorslaer, (2001), Williams and Cookson (2001), Roemer (2002), Bleichrodt et al. (2004)). The word "equity" usually refers to the distributive justice in the allocation of a commodity ("health" in this case). The underlying motivation in most of the above-mentioned literature is the so-called 'just compensation principle' by which health inequalities that are not attributable to an individual's responsibility should be compensated by society. An obvious instance of health inequalities that are not attributable to an individual's responsibility are those inequalities due to congenital impairments. The 'just compensation principle' implies that society should do its best to alleviate the consequences of congenital diseases in impaired infants.

There are some congenital diseases whose negative consequences could be alleviated by means of an early detection and a subsequent treatment. Typical examples of diseases obeying this axiom are, for instance, congenital hearing impairment, hipotiroidism or phenylcetonuria. In these cases, the implementation of an early detection protocol seems to be sufficiently justified on the sole basis of the 'just compensation principle'. Indeed, early detection protocols can be deemed as an efficient tool to avoid non-feasible future compensations to individuals suffering from congenital impairments.

If the decision about the implementation of an early detection protocol appears to be unquestionable, the selection of a particular protocol among the alternatives to be implemented is, by no means, a straightforward decision. Usually, this decision is conducted by an economic evaluation of the existing alternatives. The economic evaluation of health care programs involves both technical and value judgements. This, and the special nature of the commodity being considered (health), is the germ of the complexity 
of this problem whose multiple angles leave room for new techniques to be developed with which we can face the problem. In this paper we present one of such new techniques to address the evaluation of early detection protocols, also known as screening programs.

Screening is traditionally defined as testing a population of asymptomatic individuals to identify precursors of a disease. The subjects who test positive are sent on for further evaluation in a subsequent diagnostic evaluation to determine whether they do, in fact, have the disease. An implicit assumption underlying the clinical interest of screening programs is that early detection, before the development of symptoms, will lead to a more favorable prognosis. This is so because, by means of a screening, it is possible to treat the disease before it becomes clinically manifest, which is more effective than a later treatment. Usually, there are different screening strategies for a given disease. As mentioned above, we assume that the decision about implementing one of them is taken up exogenously and the issue is to select the best strategy to carry out, among the available ones.

The first problem that one has to face in order to run an economic evaluation of screening programs is the outcome measurement of the available strategies. This is a major issue in the discipline of health economics, since no measure has presented itself free of shortcomings and clearly superior to the other existing ones. In a companion paper, Herrero \& Moreno-Ternero (2003), we analyze the problem of selecting among screening programs, making use of the QALY measurement. The QALY is possibly the most frequently employed measure in health economics (e.g., Gold et al. (1996), Drummond et al. (1997), Dolan (2001)). It is a quite tractable measure and therefore easy to use. Nevertheless, it is both practically and conceptually dubious. Among other things, it relies on very restrictive assumptions on individual preferences. It has also been argued by many authors (e.g., Wagstaff (1991), Dolan (1998), Østerdal (2003), Bleichrodt et al. (2004)) that this index may fail to capture distributive justice.

Here we propose evaluating newborn screening programs for a given congenital disease by means of an opportunity analysis. By opportunity analysis we mean computing the opportunities a newborn screening program offers to a randomly given individual and selecting the best option accordingly. 
To formalize our model we make use of a metric of opportunity. ${ }^{1}$ In other words, we associate with each individual a unique number on a zero-one scale, interpreting that number as the degree of the potential opportunities the individual enjoys. This numerical measurement renders the subsequent analysis very tractable analytically. For a given cohort of newborns susceptible of suffering a disease, we distinguish four reference groups.

1. True positives: Those impaired infants that are detected thanks to a newborn screening program.

2. False positives: Those healthy infants that are (erroneously) identified as impaired infants, with a newborn screening program.

3. True negatives: Those healthy infants with a negative result in a newborn screening program.

4. False negatives: Those impaired infants that are not detected thanks to a screening program.

If we assume that individual opportunities do not vary within these four subgroups, but they might differ between them, we can consider as the opportunities a newborn screening program offers, the aggregation of the opportunities of an individual in each of these groups, weighted by the probability of being in each group. The reason why each screening program is associated with a different magnitude of overall potential opportunities in the population is that the previous probabilities depend on the intrinsic properties of the screening program being considered. If we adopt as the 'status quo' the absence of screening, we may identify as the outcome of a screening program the potential opportunity gains that offers, with respect to the status quo.

We formalize this model in the paper and show that the opportunity analysis just described can be considerably simplified under some mild assumptions: opportunities of true positives are strictly larger than those of false negatives (with a constant difference across screening programs) and opportunities of true negatives and of false positives are identical. Under such

\footnotetext{
${ }^{1}$ For reviews of the literature concerning metric of opportunity see, for instance, Peragine (1998) or Sugden (1998).
} 
plausible assumptions, the outcome of a program can be seen as its level of sensitivity, i.e., the probability of finding by the screening procedure a disability when it is actually there. In particular, it is interesting to note that the conclusions a opportunity analysis offers do not depend on the metric of opportunity that we choose.

One might argue that the model just described is too simplistic as other circumstances (e.g., gender, race, parental socioeconomic status, etc.) that may influence future opportunities of newborns are not considered. The next step of the paper is that of enriching the model described above by introducing individual circumstances. We develop this more complex model and remark that the previous preliminary model, simple that it is, provides us with very useful insights to solve this more general one. Indeed, we show that an additional assumption to the ones mentioned above; namely, the probability of being a true positive does not depend on the remaining individual circumstances, suffices to obtain the same conclusion: the opportunity analysis is robust to changes of the metric of opportunity.

We conclude the paper applying our model to the current debate on the implementation of newborn hearing screening programs in some states of the United States and in some European countries (e.g., National Institutes of Health Consensus Development Conference Statement, 1993; European Consensus Project on Neonatal Hearing Screening, 1999; Joint Committee on Infant Hearing, 2000; Keren et al., 2002). We show that, according to an opportunity analysis, universal programs are preferred to selective programs, in which only newborns with risk factor are screened. This conclusion agrees with the more recent pediatric recommendations that have been published (e.g., Joint Committee on Infant Hearing, 2000).

The rest of the paper is organized as follows. Section 2 contains the basic model of the opportunity analysis. The results for this model are presented in Section 3. In Section 4 we study the extended model. An application to the case of congenital hearing impairment is taken up in Section 5, and Section 6 concludes. 


\section{The model}

We consider a particular congenital impairment for which there exist newborn screening programs that permit its early detection. We assume that the early detection of the disease, followed by an adequate treatment, might reduce considerably its negative consequences. Let $\mathcal{N}=\{1, \ldots, n\}$ be the corresponding cohort of newborns susceptible of suffering the disease. The status of a newborn with respect to the disease is either $d=0$ (if the infant is healthy) or $d=1$ (if the infant is impaired). We denote by $\rho \in[0,1]$ the prevalence of the disease in the cohort, i.e., the fraction of impaired newborns in the cohort. ${ }^{2}$ We denote by $G_{0}$ the set of newborns with negative disease status and by $G_{1}$ the set of newborns with positive disease status. By construction, $\mathcal{N}=G_{0} \cup G_{1}$ and the number of newborns in each of the subgroups is $\left|G_{0}\right|=(1-\rho) \cdot n$ and $\left|G_{1}\right|=\rho \cdot n$, respectively.

Newborns can be partitioned into four groups, according to whether they do or do not have the disease and whether their screening tests are positive or negative. Thus, there are four groups of newborns: true positives, newborns whom the screening correctly indicates to have the disease; false positives, those who do not have the disease but who have a positive screening test; false negatives, those who have the disease but are mistakenly cleared by the screening; and true negatives, those who do not have the disease and are correctly identified as such by the screening. ${ }^{3}$ We can compute how likely an individual would belong to each of the four groups by using characteristics of the population (prevalence) and of the detection ability of the screening test (sensitivity and specificity). The sensitivity of the screening test $\left(\pi_{1}\right)$ is the conditional probability that an individual with the disease is positively detected by the test. This is estimated by the ratio of true positives to total impaired individuals $\left(\frac{\alpha}{\alpha+\gamma}\right)$. The specificity of the test $\left(\pi_{2}\right)$ is the conditional probability of an individual without the disease being correctly detected as negative in the test. This is measured by the ratio of true negatives to the number of disease-free individuals $\left(\frac{\delta}{\beta+\delta}\right)$. Using these definitions, the

\footnotetext{
${ }^{2}$ If $i$ denotes the number of impaired newborns in $\mathcal{N}$ then $\rho=\frac{i}{n}$. We interpret this number as the probability of a newborn in the cohort being impaired.

${ }^{3}$ If there is no screening program being implemented then the group of false negatives is $G_{1}$, whilst $G_{0}$ is the group of true negatives.
} 
probability of an individual being a true negative is the probability that she does not have the disease $(1-\rho)$ times the probability that the screening correctly indicates that she does not have the disease $\left(\pi_{2}\right)$. The probabilities of the individual to be a true positive $\left(\rho \pi_{1}\right)$, a false positive $\left((1-\rho)\left(1-\pi_{2}\right)\right)$ and a false negative $\left(\rho\left(1-\pi_{1}\right)\right)$ can be similarly expressed. The advantage of this way of writing the screening probabilities is that it makes easier to assess the implications of variations in the parameters $\rho, \pi_{1}$ or $\pi_{2}$ separately.

\section{Table 1}

Impairment status and test results

\begin{tabular}{lccc}
\hline & \multicolumn{3}{c}{ Status: } \\
\cline { 2 - 3 } Test & Positive & Negative & Total \\
\hline Positive & $\alpha$ & $\beta$ & $\alpha+\beta$ \\
Negative & $\gamma$ & $\delta$ & $\gamma+\delta$ \\
Total & $\alpha+\gamma$ & $\beta+\delta$ & $\alpha+\beta+\gamma+\delta=n$ \\
Prevalence & $\rho=\frac{\alpha+\gamma}{n}$ & & \\
\hline
\end{tabular}

Test Characteristics

\begin{tabular}{ll}
\hline Sensitivity & $\pi_{1}=\frac{\alpha}{\alpha+\gamma}$ \\
Specificity & $\pi_{2}=\frac{d}{\beta+\delta}$ \\
\hline
\end{tabular}

Let $\mathcal{S}=\left\{s^{1}, \ldots, s^{m}\right\}$ denote the set of available screening programs for the early detection of the disease. Let $s^{0}$ denote the 'status quo', i.e., the scenario without any screening program. For all $j=0,1, \ldots, m$, each screening program $s^{j}$ is defined as $s^{j}=s^{j}\left(\pi_{1}^{j}, \pi_{2}^{j}, c^{j}\right)$, where $\pi_{1}^{j}$ and $\pi_{2}^{j}$ denote the sensitivity and the specificity of $s^{j}$ respectively, and $c^{j}$ denotes the incremental costs of the screening program with respect to the status quo. ${ }^{4}$ By costs of a screening program, we mean the costs incurred by the test, i.e., technology and wages of the specialists who supervise it, and the costs of the final diagnostic evaluation to which every positive infant is referred after the screening test. It is worth noting that we assume all impaired individuals receive diagnostic evaluation, regardless of whether their impairment is detected early

\footnotetext{
${ }^{4}>$ From here onwards, unless otherwise stated, it is assumed that all costs are per capita.
} 
or not. Consequently, the incremental health-care cost of implementing a screening program $s^{j}$ is

$$
c^{j}=c_{s}^{j}+r \cdot(1-\rho) \cdot\left(1-\pi_{2}^{j}\right) \cdot c_{d},
$$

where $c_{s}^{j}$ is the cost of the screening itself, $r$ is the return rate and $c_{d}$ the cost of the diagnostic evaluation. ${ }^{5}$

For ease in exposition, denote an infant's test result in the screening program $s^{j}$ as $t^{j}=0$ if it is negative, and as $t^{j}=1$ if it is positive. Then, for each $s^{j} \in \mathcal{S}, d \in\{0,1\}$ and $t^{j} \in\{0,1\}$ denote by $G_{(d, t)}^{j}$ the group of infants sharing disease status $d$ and test result $t^{j}$, after implementing $s^{j}$. Thus, $\mathcal{N}$ is expressed as follows:

$$
\mathcal{N}=G_{(1,1)}^{j} \cup G_{(0,1)}^{j} \cup G_{(1,0)}^{j} \cup G_{(0,0)}^{j} .
$$

According to the notation introduced above, it is straightforward to see that the probabilities of being in each of the groups are given by:

$$
\begin{aligned}
\rho_{(1,1)}^{j} & =\rho \cdot \pi_{1}^{j} \\
\rho_{(0,1)}^{j} & =(1-\rho) \cdot\left(1-\pi_{2}^{j}\right) \\
\rho_{(1,0)}^{j} & =\rho \cdot\left(1-\pi_{1}^{j}\right) \\
\rho_{(0,0)}^{j} & =(1-\rho) \cdot \pi_{2}^{j}
\end{aligned}
$$

Now, we compute the potential opportunities of each newborn. To do so, we need an metric of opportunity. A metric of opportunity is a mapping associating with each individual a unique number on a zero-one scale representing the degree of potential opportunities, and interpreting 0 (1) as the lowest (highest) possible degree of potential opportunities an individual might face. Formally, a metric of opportunity is a function

$$
\Omega:\{0,1\} \times\{0,1\} \times \mathcal{S} \cup\left\{s^{0}\right\} \mapsto[0,1]
$$

where $\Omega\left(d, t, s^{j}\right) \in[0,1]$ denotes the degree of potential opportunities of an individual with disease status $d$ and a test result $t^{j}$ after implementing $s^{j}$.

\footnotetext{
${ }^{5}$ By return rate we mean the percentage of infants returned for follow-up testing.
} 
We define the degree of opportunity associated with each screening procedure $s^{j} \in \mathcal{S}$ as

$$
\Omega^{j}=\sum_{d, t=0}^{1} \rho_{(d, t)}^{j} \cdot \Omega\left(d, t, s^{j}\right)
$$

where $\rho_{(d, t)}^{j}$ is the probability of being in $G_{(d, t)}^{j}$. In other words, $\Omega^{j}$ is the sum of the degrees of potential opportunities associated with each group (true positives, false positives, false positives and true negatives) multiplied by the probability of an individual being in the group. In this respect, $\Omega^{j}$ can be interpreted as the expected opportunities of an infant after implementing $s^{j}$.

In particular, the degree of opportunity associated with the 'status quo' comes determined by

$$
\Omega^{0}=\rho \cdot \Omega\left(1,0, s^{0}\right)+(1-\rho) \cdot \Omega\left(0,0, s^{0}\right) .
$$

Consequently, the degree of opportunity gained, associated with a screening $s^{j} \in \mathcal{S}$, is $\mathcal{O}_{\Omega}^{j}=\Omega^{j}-\Omega^{0}$.

The model just described provides us with a simple framework to choose among the available screening programs by means of an opportunity analysis. As a first stage, the available programs are ranked according to the opportunities they offer. As a second stage, for a fixed stock of resources, the health authority should choose the program that provides the highest degree of opportunity gained within the set of feasible options, i.e., within those whose cost is below the available budget. If financing is not an issue we would simply advocate for implementing the program exhibiting the highest magnitude of $\mathcal{O}_{\Omega}^{j}$.

\section{A first result}

In this section we provide additional assumptions under which the opportunity analysis described in Section 2 is independent of the metric of opportunity.

The first assumption says, roughly, that opportunities do not decrease "per se' by being referred to a screening program. In other words, the degree of 
potential opportunities of a true (false) negative individual after implementing a screening program coincides with the degree of potential opportunities of a healthy (impaired) individual in the status quo. Formally:

Assumption 1: For each metric of opportunity $\Omega$ and for all $s^{j} \in \mathcal{S}$ we have

$$
\Omega\left(0,0, s^{j}\right)=\Omega\left(0,0, s^{0}\right) \text {, and } \Omega\left(1,0, s^{j}\right)=\Omega\left(1,0, s^{0}\right) \text {. }
$$

The second assumption says that there are no differences in potential opportunities between healthy individuals with different test results, i.e., between a false positive and a true negative individual. Formally:

Assumption 2: For each metric of opportunity $\Omega$ and for all $s^{j} \in \mathcal{S}$ we have

$$
\Omega\left(0,1, s^{j}\right)=\Omega\left(0,0, s^{j}\right) .
$$

The third assumption says that early detection of the disease is advantageous at an individual level, and that this individual improvement is independent of the screening method chosen. That is, the degree of opportunity gained by an impaired infant after being detected by a screening program is strictly positive and constant (although depending on the metric of opportunity) for each program. Formally:

Assumption 3: For each metric of opportunity $\Omega$ and for all $s^{j} \in \mathcal{S}$ we have

$$
\Omega\left(1,1, s^{j}\right)-\Omega\left(1,0, s^{0}\right)=\lambda(\Omega)>0 .
$$

We have the following result.

Theorem 1 Under Assumptions 1, 2 and 3, the degree of opportunity gained that a screening program offers is its sensitivity, up to a (multiplicative) constant factor.

Proof. Let $\Omega$ be a metric of opportunity. By (4), the degree of opportunity associated with the 'status quo', according to $\Omega$, is

$$
\Omega^{0}=\rho \cdot \Omega\left(1,0, s^{0}\right)+(1-\rho) \cdot \Omega\left(0,0, s^{0}\right) .
$$


Similarly, by (3), given a screening procedure $s^{j} \in \mathcal{S}$, its degree of opportunity according to $\Omega$ is

$$
\Omega^{j}=\sum_{d, t=0}^{1} \rho_{(d, t)}^{j} \cdot \Omega\left(d, t, s^{j}\right) .
$$

Since for every $d \in\{0,1\}$ and $s^{j} \in \mathcal{S}, G_{(d, 0)}^{j}$ and $G_{(d, 1)}^{j}$ are disjoint sets, then $\rho=\rho_{(1,0)}^{j}+\rho_{(1,1)}^{j}$ and $1-\rho=\rho_{(0,0)}^{j}+\rho_{(0,1)}^{j}$. Thus,

$$
\Omega^{0}=\left(\rho_{(1,0)}^{j}+\rho_{(1,1)}^{j}\right) \cdot \Omega\left(1,0, s^{0}\right)+\left(\rho_{(0,0)}^{j}+\rho_{(0,1)}^{j}\right) \cdot \Omega\left(0,0, s^{0}\right) .
$$

Consequently, the degree of opportunity gained that $s^{j}$ offers is:

$$
\begin{aligned}
\mathcal{O}_{\Omega}^{j}= & \Omega^{j}-\Omega^{0} \\
= & \rho_{(0,0)}^{j} \cdot\left(\Omega\left(0,0, s^{j}\right)-\Omega\left(0,0, s^{0}\right)\right)+\rho_{(1,0)}^{j} \cdot\left(\Omega\left(1,0, s^{j}\right)-\Omega\left(1,0, s^{0}\right)\right)+ \\
& \rho_{(0,1)}^{j} \cdot\left(\Omega\left(0,1, s^{j}\right)-\Omega\left(0,0, s^{0}\right)\right)+\rho_{(1,1)}^{j} \cdot\left(\Omega\left(1,1, s^{j}\right)-\Omega\left(1,0, s^{0}\right)\right)
\end{aligned}
$$

By Assumptions 1 and 2 we have the following:

$$
\mathcal{O}_{\Omega}^{j}=\rho_{(0,0)}^{j} \cdot\left(\Omega\left(1,1, s^{j}\right)-\Omega\left(1,0, s^{0}\right)\right)=\rho \cdot \pi_{1}^{j} \cdot\left(\Omega\left(1,1, s^{j}\right)-\Omega\left(1,0, s^{0}\right)\right) .
$$

By Assumption $3, \lambda(\Omega)=\Omega\left(1,1, s^{j}\right)-\Omega\left(1,0, s^{0}\right)>0$, for all $s^{j} \in \mathcal{S}$. Then,

$$
\mathcal{O}_{\Omega}^{j}=k \cdot \pi_{1}^{j},
$$

where

$$
k=k(\rho, \Omega)=\rho \cdot \lambda(\Omega)>0 .
$$

Note that $k$ depends on the prevalence of the impairment and the metric of opportunity. It is not, however, screening method-specific.

The main relevance of Theorem 1 lies on the fact that, under Assumptions 1 to 3 , the decision about the programs, according to the opportunity analysis we described in Section 2, does not depend on the metric of opportunity that we decide to fix. 
It can be inferred from the proof of the theorem that both the degrees of opportunity gained and the sensitivity levels of the programs yield the same ranking of preferences among the set of alternative programs. The cardinal information of these preferences is captured by the constant $k$ that appears in the proof, which depends on the prevalence of the impairment and the metric of opportunity.

To conclude, we acknowledge that, since the prevalence of the disease appears in the constant factor $k$, our result is only informative when the analysis refers to screening programs for the same disease. It cannot be used, however, to compare screening programs of different diseases.

\section{Including circumstances}

One might argue that the model described above is very simple. In particular, it only computes the influence of suffering a congenital disease on the potential opportunities of a newborn and rules out any other. Suffering a congenital disease is definitely a circumstance, i.e., a factor beyond the control of an individual, that affects the potential opportunities of an individual. Surely, there are many others that also affect, positively or negatively. Instances are the gender, the race, the parental socioeconomic status, the level of formal education attained by their parents, and so on. The next step of the paper is enriching the model described above by introducing individual circumstances.

Assume each infant in the cohort $\mathcal{N}$ is identified by a profile of individual circumstances, the disease status being one of them. We denote by $\mathcal{C}$ the set of possible sub-profiles of circumstances, that do not comprise the disease status $d$. For each profile of circumstances $(c, d) \in \mathcal{C} \times\{0,1\}$, we denote by $G_{(c, d)}$ the group of newborns that share that profile. Then,

$$
\mathcal{N}=\bigcup_{c \in \mathcal{C}} \bigcup_{d \in\{0,1\}} G_{(c, d)},
$$

where $G_{(c, d)} \cap G_{(c, d)}=\emptyset$, for all $(c, d) \neq\left(c^{\prime}, d^{\prime}\right)$ in $\mathcal{C} \times\{0,1\}$. For each $(c, d) \in \mathcal{C} \times\{0,1\}$, we denote by $\rho_{(c, d)}$ the probability of being in $G_{(c, d)} .{ }^{6}$ Then,

\footnotetext{
${ }^{6}$ More precisely, $p_{(c, d)}=\frac{\left|G_{(c, d)}\right|}{q}$, where $\left|G_{(c, d)}\right|$ denotes the cardinality of $G_{(c, d)}$.
} 
$\rho=\sum_{c \in \mathcal{C}} \rho_{(c, 1)}$. Finally, for the sake of completeness, let $\rho_{c}=\rho_{(c, 0)}+\rho_{(c, 1)}$ be the probability of sharing the sub-profile of circumstances $c$.

After the implementation of a screening program, there is a subsequent and new population partition, depending on the test results and the circumstances. If, for each $s^{j} \in \mathcal{S}, c \in \mathcal{C}, d \in\{0,1\}$ and $t^{j} \in\{0,1\}$ we denote by $G_{(c, d, t)}^{j}$ the group of infants sharing circumstances $c$, disease status $d$ and test result $t^{j}$, after implementing $s^{j}$, then $\mathcal{N}$ is expressed as follows:

$$
\mathcal{N}=\bigcup_{c \in \mathcal{C}} \bigcup_{d \in\{0,1\}} \bigcup_{t \in\{0,1\}} G_{(c, d, t)}^{j} .
$$

Recall from Section 2 that $\rho_{(d, t)}^{j}$ denotes the probability of being in $G_{(d, t)}^{j}$, i.e., with disease status $d$ and test result $t^{j}$ after implementing $s^{j}$. If $\rho_{(c, d, t)}^{j}$ denotes the probability of being in the group $G_{(c, d, t)}^{j}$ then $\rho_{(d, t)}^{j}=\sum_{c \in \mathcal{C}} \rho_{(c, d, t)}^{j}$.

In this new framework, a metric of opportunity is a mapping associating with each profile of circumstances and test result a unique number on a zeroone scale representing the degree of potential opportunities, and interpreting 0 (1) as the lowest (highest) possible degree of potential opportunities an individual might face. Formally, a function

$$
\Omega: \mathcal{C} \times\{0,1\} \times\{0,1\} \times \mathcal{S} \cup\left\{s^{0}\right\} \mapsto[0,1],
$$

where $\Omega\left(c, d, t, s^{j}\right) \in[0,1]$ denotes the degree of potential opportunities of an individual with profile of circumstances $(c, d)$ and a test result $t^{j}$ after implementing $s^{j}$.

The degree of opportunity gained, associated with a screening $s^{j} \in \mathcal{S}$, is defined in an analogous manner to that of Section 3, i.e., $\mathcal{O}_{\Omega}^{j}=\Omega^{j}-\Omega^{0}$, where now

$$
\Omega^{j}=\sum_{c \in \mathcal{C}} \sum_{d, t=0}^{1} \rho_{(c, d, t)}^{j} \cdot \Omega\left(c, d, t, s^{j}\right) .
$$

for each $s^{j} \in \mathcal{S} \cup\left\{s^{0}\right\}$.

We now mimic the assumptions of Section 3 into this framework.

Assumption 1: For each metric of opportunity $\Omega$, for all $s^{j} \in \mathcal{S}$, and for all $c \in \mathcal{C}$ we have

$$
\Omega\left(c, 0,0, s^{j}\right)=\Omega\left(c, 0,0, s^{0}\right), \text { and } \Omega\left(c, 1,0, s^{j}\right)=\Omega\left(c, 1,0, s^{0}\right) .
$$


Assumption 2: For each metric of opportunity $\Omega$, for all $s^{j} \in \mathcal{S}$, and for all $c \in \mathcal{C}$ we have

$$
\Omega\left(c, 0,1, s^{j}\right)=\Omega\left(c, 0,0, s^{j}\right)
$$

Assumption 3: For each metric of opportunity $\Omega$, for all $s^{j} \in \mathcal{S}$, and for all $c \in \mathcal{C}$ we have

$$
\Omega\left(c, 1,1, s^{j}\right)-\Omega\left(c, 1,0, s^{0}\right)=\lambda(\Omega, c)>0 .
$$

The plausibility of these three assumptions seems to be unobjectionable.

We include now a fourth assumption whose plausibility depends on the particular framework that we consider. It says that the probability of being a true positive does not depend on the individual sub-profile of circumstances. In other words, two newborns with different sub-profile of circumstances $c_{1}, c_{2} \in \mathcal{C}$ have the same probability of being true positives. In order to formalize this assumption, we introduce a piece of notation. For $x \in \mathcal{N}$ denote by $c(x)$ her sub-profile of circumstances. Then, the assumption is the following:

Assumption 4: For all $x \in \mathcal{N}, s^{j} \in \mathcal{S}$, and $c \in \mathcal{C}$ we have

$$
\operatorname{Pr}\left(x \in G_{(1,1)}^{j} \mid c(x)=c\right)=\operatorname{Pr}\left(x \in G_{(1,1)}^{j}\right) .
$$

Note that this assumption is less strong than what one might initially think. Assumption 4 is not saying that the impairment status of an infant and the remaining circumstances are uncorrelated. It is saying however, that being identified as a true positive will not depend on the remaining circumstances.

We obtain the following result.

Theorem 2 Under Assumptions 1, 2, 3 and 4, the degree of opportunity gained that a screening program offers is its sensitivity, up to a (multiplicative) constant factor. 
Proof. Let $\Omega$ be a metric of opportunity. By (6), the degree of opportunity associated with the 'status quo', according to $\Omega$, is

$$
\Omega^{0}=\sum_{c \in \mathcal{C}}\left(\rho_{(c, 0)} \cdot \Omega\left(c, 0,0, s^{0}\right)+\rho_{(c, 1)} \cdot \Omega\left(c, 1,0, s^{0}\right)\right) .
$$

Similarly, given a screening procedure $s^{j} \in \mathcal{S}$, its degree of opportunity according to $\Omega$ is

$$
\Omega^{j}=\sum_{c \in \mathcal{C}} \sum_{d, t=0}^{1} \rho_{(c, d, t)}^{j} \cdot \Omega\left(c, d, t, s^{j}\right) .
$$

Thus, the degree of opportunity gained that $s^{j}$ offers is:

$\mathcal{O}_{\Omega}^{j}=\Omega^{j}-\Omega^{0}=\sum_{c \in \mathcal{C}} \sum_{d \in\{0,1\}}\left(\rho_{(c, d, 0)}^{j} \cdot \Omega\left(c, d, 0, s^{j}\right)+\rho_{(c, d, 1)}^{j} \cdot \Omega\left(c, d, 1, s^{j}\right)-\rho_{(c, d)} \cdot \Omega\left(c, d, 0, s^{0}\right)\right)$

Since for every $c \in \mathcal{C}, d \in\{0,1\}$ and $s^{j} \in \mathcal{S}, G_{(c, d, 0)}^{j}$ and $G_{(c, d, 1)}^{j}$ are disjoint sets and $G_{(c, d)}=G_{(c, d, 0)}^{j} \cap G_{(c, d, 1)}^{j}$, then $\rho_{(c, d)}=\rho_{(c, d, 0)}^{j}+\rho_{(c, d, 1)}^{j}$. Thus,

$$
\begin{aligned}
\mathcal{O}_{\Omega}^{j}= & \sum_{c \in \mathcal{C}} \rho_{(c, 0,0)}^{j} \cdot\left(\Omega\left(c, 0,0, s^{j}\right)-\Omega\left(c, 0,0, s^{0}\right)\right)+ \\
& \sum_{c \in \mathcal{C}} \rho_{(c, 1,0)}^{j} \cdot\left(\Omega\left(c, 1,0, s^{j}\right)-\Omega\left(c, 1,0, s^{0}\right)\right)+ \\
& \sum_{c \in \mathcal{C}} \rho_{(c, 0,1)}^{j} \cdot\left(\Omega\left(c, 0,1, s^{j}\right)-\Omega\left(c, 0,0, s^{0}\right)\right)+ \\
& \sum_{c \in \mathcal{C}} \rho_{(c, 1,1)}^{j} \cdot\left(\Omega\left(c, 1,1, s^{j}\right)-\Omega\left(c, 1,0, s^{0}\right)\right) .
\end{aligned}
$$

By Assumptions 1, 2 and 3,

$$
\mathcal{O}_{\Omega}^{j}=\sum_{c \in \mathcal{C}} \rho_{(c, 1,1)}^{j} \cdot \lambda(\Omega, c),
$$

where $\lambda(\Omega, c)=\Omega\left(c, 1,1, s^{j}\right)-\Omega\left(c, 1,0, s^{0}\right)>0$. Now, by the definition of conditional probability, $\rho_{(c, 1,1)}^{j}=\rho_{c} \cdot \operatorname{Pr}\left(x \in G_{(1,1)}^{j} \mid c(x)=c\right)$. By Assumption $4, \operatorname{Pr}\left(x \in G_{(1,1)}^{j} \mid c(x)=c\right)=\operatorname{Pr}\left(x \in G_{(1,1)}^{j}\right)$. Since $\operatorname{Pr}\left(x \in G_{(1,1)}^{j}\right)=\rho_{(1,1)}^{j}=$ $\rho \cdot \pi_{1}^{j}$, it follows that

$$
\mathcal{O}_{\Omega}^{j}=k \cdot \pi_{1}^{j}
$$


where

$$
k=k\left(\mathcal{C}, \rho, \rho_{c}, \Omega\right)=\rho \cdot \sum_{c \in \mathcal{C}} \rho_{c} \cdot \lambda(\Omega, c)>0
$$

Note that $k$ depends on the set of circumstances $(\mathcal{C})$, the prevalence of the impairment $(\rho)$, the probabilities of each profile of circumstances $\left(\rho_{c}\right)$ and the metric of opportunity $(\Omega)$. It is not screening method-specific, however.

Theorem 2 mimics Theorem 1 in a more general framework where individual circumstances are also considered to compute individual potential opportunities. The conclusion both theorems offer is the same, namely, the opportunity analysis of newborn screening programs does not depend on the metric of opportunity that we decide to fix. More precisely, they show that both the degrees of opportunity gained and the sensitivity levels of the programs yield the same ranking of preferences among the set of alternative programs. The only price we have to pay to obtain the same result in the more general model is that of accepting Assumption 4. As mentioned above, the plausibility of this assumption depends on the particular framework that we consider.

\section{Application: the case of congenital hearing impairment}

We conclude by applying our model to the case of congenital hearing impairment. This is a disease that satisfies all the medical requirements to impose a prevention program, based on a newborn screening protocol. First of all, it is a serious disease, for which a lack of early diagnosis will cause problems in language acquisition. Significant hearing loss interferes with the development of speech perception abilities needed for later language learning (Yoshinaga-Itano et al., 1999). These impairments in communication skills can lead to learning disabilities and ultimately, to limitations in career opportunities. Moreover, it is more frequent than other impairments for which newborn screening programs are in use in developed countries (White and 
Maxon, 1995). Finally, there are reliable screening methods, with high levels of sensitivity and specificity, and there is also an effective treatment available.

Due to these facts, there is a broad agreement to impose a newborn hearing screening program, as subsequently recommended the National Institutes of Health Consensus Statement on the Early Identification of Hearing Impairment in Infants and Young Children, 1993; the European Consensus Statement on Neonatal Hearing Screening, 1999; and the Statement of the Joint Committee on Infant Hearing, 2000.

Having reached this consensus, the debate moved to select between a universal and a selective alternative. In a Universal Newborn Hearing Screening ( "UNHS" hereafter) every newborn is tested, whereas in a Selective Newborn Hearing Screening ("SNHS" hereafter) only those who were born with a risk factor, such as being in the neonatal intensive care unit or having a family history of hearing impairment, are tested. A UNHS is more expensive but also more effective, since only $50 \%$ of newborns with a hearing impairment belong to a group at risk (National Institutes of Health Consensus Development Conference Statement, 1993). It is currently mandated in 32 states of the United States (Keren et al., 2002). The SNHS, however, was and continues to be practiced throughout the United States and the rest of the world (Keren et al., 2002).

There is ample literature on choosing between UNHS and SNHS, especially from the medical viewpoint (e.g., National Institutes of Health Consensus Development Conference Statement, 1993; Bess and Paradise, 1994; Downs and Yoshinaga-Itano, 1999; European Consensus Project on Neonatal Hearing Screening, 1999; Joint Committee on Infant Hearing, 2000, Thompson et al., 2001), but also from an economic viewpoint (e.g., Kemper and Downs, 2000; Kezirian et al., 2001; Keren et al., 2002; Herrero and MorenoTernero, 2004). The aim of this section is to apply our model to provide an additional viewpoint to this current debate about choosing between the two alternatives. 


\subsection{Protocols}

According to the recommendations of the Joint Committee on Infant Hearing, every neonate should be tested by Otoacoustic Emissions ( "OAE" hereafter), a less efficient and expensive test, followed by Auditory Brainstem Responses ( " $A B R$ " hereafter), a more efficient and expensive test, for those who failed the initial stage (Joint Committee on Infant Hearing, 2000). We evaluate two slightly different versions of this universal 2-stage screening, by changing some aspects of the OAE and ABR strategies. On the one hand, we consider automated transient-evoked otoacoustic emissions (TEOAE) as a first stage, followed, when indicated, by automated auditory brainstem response (AABR) testing. We refer to this protocol as U1. On the other hand, we consider otoacoustic emissions (OAE) as a first stage, followed, when indicated, by a shorter screening version of automated auditory brain response testing (S-ABR). We refer to this protocol as U2. ${ }^{7}$ Finally, there is a different UNHS currently in practice in a Spanish region (Navarra). In this case, the protocol has three stages. The first stage consists on an OAE test to every newborn at the third day of life, before leaving the nursery. For those who failed it, there will be a second OAE at the fifteenth day of life. Finally, the third stage involves a new OAE test for those neonates who failed the second stage and return at the third month. We will refer to this protocol as U3.

A selective screening includes a previous stage with a high-risk criterion (HRC), and then applies the protocol for infants at risk for congenital hearing loss. We therefore have three alternative selective screening procedures, which will be called S1, S2 and S3. Each protocol (selective or universal) concludes with a diagnostic evaluation for those who failed after the last stage.

To summarize, we focus our attention on six alternative early detection programs. Formally, following the notation of Section 2, let $s^{0}$ denote the absence of a screening procedure, $s_{1}\left(s_{2}\right)\left[s_{3}\right]$ the first (second) [third] UNHS procedure, and $s_{4}\left(s_{5}\right)\left[s_{6}\right]$ the first (second) [third] SNHS, based on high risk

\footnotetext{
${ }^{7}$ The reader is referred to Kemper \& Downs (2000) and Kezirian et al. (2001) for further details about protocols U1 and U2.
} 
factors.

Table 2

Data of the screening procedures

\begin{tabular}{cccccccc}
\hline & \multicolumn{7}{c}{ Screening } \\
\cline { 2 - 8 } Parameters & $s^{0}$ & $s^{1}$ & $s^{2}$ & $s^{3}$ & $s^{4}$ & $s^{5}$ & $s^{6}$ \\
\hline Sensitivity $\left(\pi_{1}^{j}\right)$ & 0 & .784 & .902 & .840 & .463 & .532 & .496 \\
Specificity $\left(\pi_{2}^{j}\right)$ & 1 & .996 & .950 & .995 & .999 & .998 & .999 \\
Direct cost $\left(c^{j}\right)$ & 0 & 10.05 & 13.91 & 11.68 & 1.59 & 1.65 & 1.57 \\
\hline Prevalence $(\rho)$ & \multicolumn{7}{c}{.0011} \\
\end{tabular}

Table 2 shows the mean estimates of the general and specific data from each procedure. Additional information about such data, like their confidence intervals, can be obtained in Kemper \& Downs (2000), Kezirian et al. (2001) and Keren et al. (2002).

\subsection{Opportunity Analysis}

Now, we provide an additional viewpoint to this debate by means of an opportunity analysis. Apart from the congenital hearing impairment status, we consider the gender as an additional circumstance. There is no reported evidence against Assumption 4 of Section 4 in this case. The remaining assumptions of Section 4 are also sound in the framework of newborn hearing screening. Hence, the opportunity analysis can be reduced to the study of the sensitivity of each program. As a result, preferences among the alternatives, would be the following:

$$
s_{2} \succsim s_{3} \succsim s_{1} \succsim s_{5} \succsim s_{6} \succsim s_{4}
$$

Note that this is precisely the ranking that one obtains considering the whole stream of costs associated with these programs. ${ }^{8}$ In particular, this ranking says that universal programs are preferred to selective programs, as recommended by the NIH and the JCIH consensus statement.

\footnotetext{
${ }^{8}$ Although the ranking according to their direct costs is different, this is precisely what we obtain when computing indirect costs like special education or disability allowances (e.g., Herrero and Moreno-Ternero (2004)).
} 
For the sake of completeness, and to get additional information about the cardinality of the preferences over the set of alternatives, we provide an opportunity analysis for a given metric of opportunity. We might think of several metrics to deal with this task. Here, we consider the notion of degree of potential success, introduced by Mariotti (2002), that particularly fits to this example.

The basic idea provided by Mariotti is the definition of success by means of different variables, reaching some minimal values. Success could be, for instance, the attainment of a minimum level of income, or a certain level education, or even a certain life expectancy above some level of good health. The definition of success might depend, not only on the congenital impairment status, but also on other circumstances, like the gender in our case. Assume that a particular definition of success has been agreed upon. The metric of opportunity $\Omega$ is to be interpreted as the probability of reaching success.

More precisely, we assume that a healthy male will certainly reach success, no matter if he was referred to a screening program. Formally, if $m$ means 'male', then

$$
\Omega\left(m, 0,1, s^{j}\right)=\Omega\left(m, 0,0, s^{j}\right)=\Omega\left(m, 0,0, s^{0}\right)=1,
$$

for all $s^{j} \in S$. We assume, however, that for females this probability is only 0.9 , i.e.,

$$
\Omega\left(f, 0,1, s^{j}\right)=\Omega\left(f, 0,0, s^{j}\right)=\Omega\left(f, 0,0, s^{0}\right)=0.9,
$$

for all $s^{j} \in S$ and where $f$ means 'female'. Obviously, for impaired infants, probabilities are lower. Nevertheless, for those who were detected by a screening, the probabilities are slightly higher. Formally:

$$
\Omega\left(m, 1,1, s^{j}\right)=0.6>\Omega\left(m, 1,0, s^{j}\right)=\Omega\left(m, 1,0, s^{0}\right)=0.1,
$$

and

$$
\Omega\left(f, 1,1, s^{j}\right)=0.5>\Omega\left(f, 1,0, s^{j}\right)=\Omega\left(f, 1,0, s^{0}\right)=0,
$$

for all $s^{j} \in S$. It is then straightforward to show that

$$
\lambda(\Omega, m)=\Omega\left(m, 1,1, s^{j}\right)-\Omega\left(m, 1,0, s^{0}\right)=0.5,
$$


and

$$
\lambda(\Omega, f)=\Omega\left(f, 1,1, s^{j}\right)-\Omega\left(f, 1,0, s^{0}\right)=0.5 .
$$

To conclude, assume that $60 \%$ of the newborns are females, i.e., $\rho_{m}=0.4$ and $\rho_{f}=0.6$.

Under this data, it is straightforward to compute that the degree of opportunity gained each program offers is

$$
\mathcal{O}_{\Omega}^{j}=\frac{\rho}{2} \cdot \pi_{1}^{j}=0.0005 \cdot \pi_{1}^{j},
$$

where $\rho$ is the prevalence of congenital hearing loss. Table 3 shows the degree of opportunity gained each program offers.

Table 3

\begin{tabular}{ccccccc}
\hline \multicolumn{6}{c}{ Degree of opportunity gained } \\
\hline \multirow{6}{*}{ Opportunity } & $s^{1}$ & $s^{2}$ & $s^{3}$ & $s^{4}$ & $s^{5}$ & $s^{6}$ \\
\cline { 2 - 7 }$\left(\mathcal{O}_{\Omega}^{j}\right)$ & 0.00039 & $\mathbf{0 . 0 0 0 4 5}$ & 0.00042 & 0.00023 & 0.00027 & 0.00024
\end{tabular}

\section{Discussion}

In this paper, we have presented a new technique to select the best newborn screening program for a particular congenital impairment out of alternative options. Such a technique consists on evaluating screening programs by means of the potential opportunities they produce. If financing is not an issue, we would advocate for implementing the program that provides the highest potential opportunities.

Now, in health care, as in other areas of social policy, decisions have to be made concerning the allocation of scarce resources. Usually, the program which shows the highest benefits is one of the most expensive programs. For a fixed budget, the program that should be implemented is the one providing higher potential opportunities whose cost is below the budget. In general, the health authority has a public budget to be distributed among different issues, rather than a fixed budget devoted to fight against a particular disease. Thus, comparisons with other programs have to be made. 
The discipline of health economics has adopted a standard solution to address these comparisons and to solve the hypothetical trade-off between costs and benefits of health care programs. That solution obtains the ratios between the cost and the outcomes of each program, and selects the one that shows the lowest cost per unit of outcome. Under the same spirit, we could consider the cost-opportunity ratios. If the program that offers the highest potential opportunities is not the less expensive one, then we would look at the cost-opportunity ratios and would select the one with the lowest cost-opportunity ratio.

In this paper, we addressed opportunities by means of a metric of opportunity. Now, opportunities can also be interpreted in terms of capability sets (Sen, 1985; Herrero and Pinto, 2003). In a health care context, the capability set of a certain person is to be understood as the set of health profiles achievable by this person. It is not her health outcome, but rather, the set of her plausible health outcomes. The opportunities of a person increase when her capability set becomes higher. This is precisely what would happen for an impaired newborn whose impairment is detected by means of a newborn screening program. The opportunity analysis presented here could have been easily adapted and framed in terms of capability sets.

To conclude, we have applied our model to the current debate on the implementation of newborn hearing screening programs in some states of the United States and in some European countries (e.g., National Institutes of Health Consensus Development Conference Statement, 1993; European Consensus Project on Neonatal Hearing Screening, 1999; Joint Committee on Infant Hearing, 2000; Keren et al., 2002). We show that, according to an opportunity analysis, universal programs are preferred to selective programs, in which only newborns with risk factor are screened. This conclusion agrees with the recent pediatric recommendations that have been published (e.g., Joint Committee on Infant Hearing, 2000). 


\section{References}

[1] Bess FH, \& Paradise JL. (1994) Universal screening for infant hearing impairment: not simple, not risk-free, not necessarily beneficial and not presently justified. Pediatrics. 93 (2) 330-334.

[2] Bleichrodt, H. (1997) Health utility indices and equity considerations, Journal of Health Economics 16, 65-91

[3] Bleichrodt H, Diecidue E, Quiggin, J (2004) Equity weights in the allocation of health care: the rank-dependent QALY model, Journal of Health Economics 23, 157-171

[4] Byrne, M, \& Thompson P. (2001) Screening and preventable illness, Journal of Health Economics 20 1077-1088

[5] Dolan P. (1998) The measurement of individual utility and social welfare. Journal of Health Economics 17, 39-52.

[6] Dolan P. (2001) The measurement of health-related quality of life for use in resource allocation decisions. Chapter 32 in Handbook of Health Economics, A.J. Culyer and J.P. Newhouse (Eds.), North Holland. Amsterdam, The Netherlands.

[7] van Doorslaer, E. et al. (2000) Equity in the delivery of health care in Europe and the US Journal of Health Economics 19, 553-583.

[8] Downs MP, \& Yoshinaga-Itano C. (1999) The efficacy of early identification and intervention for children with hearing impairment, Pediatr. Clin. North. Am. 46 (1) 79-87.

[9] Drummond M, O'Brien BJ, Stoddart GL, Torrance GW. (1997) Methods for the economic evaluation of health care programmes. Second Edition O.U.P.

[10] European consensus project on neonatal hearing screening (1999) European consensus statement on neonatal hearing screening. Eur. J. Pediatr. 158. 95-96. 
[11] Gold, MR, Siegel, JE, Rusell, LB, Weinstein, MC. (1996) Costeffectiveness in health and medicine. Oxford University Press. New York.

[12] Grupo técnico de trabajo sobre la detección precoz de hipoacusias neonatales en Navarra (1999) Programa universal de detección precoz de hipoacusias neonatales en Navarra. Servicio de docencia, investigación y desarrollo sanitario. Departamento de salud. Govierno de Navarra.

[13] Herrero, C. \& Moreno-Ternero, J.D. (2003), A new outcome measure for cost-utility analyses of screening programs. IVIE WP-AD 2003-24.

[14] Herrero C. \& Moreno-Ternero J.D. (2004), Hospital Costs and Social Costs: A Case Study of Newborn Hearing Screening. Investigaciones Económicas. Forthcoming.

[15] Herrero, C. \& Pinto, J.L. (2003), Capabilities and opportunities in health. Mimeo.

[16] Joint Committee on Infant Hearing (2000), Year 2000 position statement: principles and guidelines for early hearing detection and intervention programs. Am. J. Audiol. 9. 9-29.

[17] Kemper, A.R. \& Downs, S.M. (2000), A cost-effectiveness analysis of newborn hearing screening strategies, Arch. Pediatr. Adolesc. Med. $154(5)$.

[18] Keren, R., Helfand, M., Homer, C.J., McPhillips, H., Lieu, T.L. (2002) Projected cost-effectiveness of statewide universal newborn hearing screening: summary of evidence. Pediatrics 110, 855-864.

[19] Kezirian, E.J., White, K.R., Yueh, B. \& Sullivan, S. (2001), Cost and cost-effectiveness of universal screening for hearing loss in newborns, Otolaryngol Head Neck Surg. 124(4). 359-67.

[20] Mariotti, M. (2002) Unequal opportunities: should the dustman's daughter have the same chances in life as the doctor's son? Mimeo. University of Exeter. 
[21] National Institutes of Health (1993), Consensus statement. Early identification of hearing impairment in infants and young children. NIH Consensus Statement. Mar 1-3. 11, 1-24.

[22] Østerdal (2003), Axioms for health care resource allocation. Mimeo. University of Copenhagen.

[23] Peragine, V. (1999) "The Distribution and Redistribution of Opportunities", Journal of Economic Surveys 13: 37-69.

[24] Roemer, J.E. (2002), Equity in health care delivery. Mimeo. Yale University.

[25] Sen, A. (1985) Commodities and Capabilities, Amsterdam. North Holland.

[26] Sugden, R. (1998) "The Metric of Opportunity" Economics and Philosophy 14: 307-37.

[27] Thompson, D.C., McPhillips, H., Davis, R.L., Lieu, T.L., Homer, C.J., Helfand, M. (2001) Universal newborn hearing screening: summary of evidence. JAMA 286, 2000-2010.

[28] Wagstaff, A. (1991) QALYs and the equity-efficiency trade-off. Journal of Health Economics 10, 21-41.

[29] Wagstaff and van Dorslaer (2001) Equity in health care finance and delivery. Chapter 34 in Handbook of Health Economics, A.J. Culyer and J.P. Newhouse (Eds.), North Holland. Amsterdam, The Netherlands.

[30] Williams and Cookson (2001) Equity in health. Chapter 35 in Handbook of Health Economics, A.J. Culyer and J.P. Newhouse (Eds.), North Holland. Amsterdam, The Netherlands.

[31] White KR, Maxon AB. (1995) Universal screening for infant hearing impairment: simple, beneficial and presently justified. Int. J. Pediatr. Otorhinolaryngol. 32 (3). 201-211. 
[32] Yoshinaga-Itano C, Sedey AL, Coulter DK, Mehl AL. (1998) Language of early and later identified children with hearing loss. Pediatrics. 102 (5) 1161-71. 